\title{
Dental Implant Outcomes in Grafted Sockets: a Systematic Review and Meta-Analysis
}

\author{
Ausra Ramanauskaite', Tiago Borges ${ }^{2}$, Bruno Leitão Almeida ${ }^{2}$, Andre Correia ${ }^{2}$ \\ ${ }^{1}$ Department of Oral Surgery and Implantology, Carolinum, Goethe University, Frankfurt, Germany. \\ ${ }^{2}$ Center for Interdisciplinary Research in Health, Institute of Health Sciences, Universidade Católica Portuguesa, Viseu, \\ Portugal.
}

\author{
Corresponding Author: \\ Ausra Ramanauskaite \\ Department of Oral Surgery and Implantology \\ Carolinum, Goethe University, Frankfurt \\ Germany \\ Phone: +496963017924 \\ Fax: +496963013829 \\ E-mail: Ramanauskaite@med.uni-frankfurt.de
}

\begin{abstract}
Objectives: To assess the treatment outcomes of the dental implants placed in the grafted sockets.

Material and Methods: A search protocol was developed to evaluate the treatment outcomes of dental implants placed in the grafted sockets in terms of implant survival rates (primary outcome), marginal-bone-level (MBL) changes, clinical parameters (i.e., bleeding on probing, probing depth), occurrence of peri-implant diseases, and aesthetic outcomes (secondary outcomes). Randomized controlled clinical trials (RCTs), controlled clinical trials, and prospective studies with at least 12 months of follow-up and a minimum of 10 patients having at least one dental implant inserted into the grafted socket were conducted. MEDLINE (PubMed) was searched for relevant articles published until $1^{\text {st }}$ April 2019. A meta-analysis was performed using the random-effects model on the selected qualifying articles.

Results: The present analysis included 7 RCTs. The survival rate of the implants inserted into the grafted sockets ranged from 95 to $100 \%$ after 1 to 4 years of follow-up. MBL loss was found to be significantly greater for the implants placed in the non-grafted healed sites than for those placed in the previously grafted sockets (weighted mean difference $=-1.961 \mathrm{~mm}, \mathrm{P}<$ 0.0001). In terms of MBL changes, no difference was detected between immediately inserted implants versus implants placed in previously grafted sockets. None of the included studies reported on the clinical parameters or occurrence of peri-implant diseases

Conclusions: Implants inserted into the previously grafted sockets showed high survival rates and lower marginal-bone-level loss than the implants inserted into the non-grafted sites.
\end{abstract}

Keywords: alveolar bone atrophy; alveolar bone grafting; dental implants; tooth socket.

\section{Accepted for publication: 5 September 2019}

To cite this article:

Ramanauskaite A, Borges T, Almeida BL, Correia A.

Dental Implant Outcomes in Grafted Sockets: a Systematic Review and Meta-Analysis

J Oral Maxillofac Res 2019;10(3):e8

URL: http://www.ejomr.org/JOMR/archives/2019/3/e8/v10n3e8.pdf

doi: $\underline{10.5037 / \text { jomr.2019.10308 }}$ 


\section{INTRODUCTION}

Extraction of a natural tooth inevitably results in significant three-dimensional alveolar bone resorption, particularly within the first 6 months $[\underline{1}, \underline{2}]$. As a consequence, alveolar ridge resorption can complicate the placement of implants into prosthetically driven positions. Therefore, to limit the post extraction dimensional changes, alveolar ridge preservation therapies, which intend to preserve the ridge volume in the envelope existing at the time of extraction, have been proposed [ $\underline{3}]$.

The results of previous systematic reviews indicate that alveolar ridge preservation is effective at minimizing bone reduction [4-6]. In particular, alveolar ridge preservation performed immediately after tooth extraction were shown to result in significantly less vertical and horizontal contraction of the alveolar crest compared to the spontaneous healing [5]. Additionally, the beneficial effect of alveolar ridge preservation was more pronounced in the prevention of horizontal bone resorption, followed by the prevention of resorption in the vertical mid-buccal and vertical mid-lingual bone $[\underline{4}, \underline{6}]$.

With regard to clinical outcomes of implants placed in grafted sites, the implant survival rates following lateral bone augmentation or sinus-floor elevation with the lateral approach were reported to be high and comparable to the ones placed into native bone $[\underline{7}, \underline{8}]$. Likewise, based on previous clinical studies, implants placed simultaneously with guided bone regeneration procedures exhibited clinical performance (with respect to survival rates, marginal-bone-height, and peri-implant soft tissue parameters) similar to that of implants in non-grafted sites [9-11]. Moreover, lateral bone augmentation procedures were found to be associated with peri-implant tissue stability after short-term (1 to 3 year) and long-term ( $>3$ year) follow-ups [12].
Until now, however, there has been limited evidence regarding the clinical outcomes of implants inserted following ridge preservation. Hence, the aim of this systematic review is to assess the existing evidence regarding the clinical outcomes of implants placed into previously grafted extraction sockets.

\section{MATERIAL AND METHODS Protocol and registration}

The methods of the analysis, inclusion and exclusion criteria were specified in advance and documented in a protocol. The review was registered in PROSPERO, an international prospective register of systematic reviews.

The review protocol was developed according to the PRISMA (Preferred Reporting Items for Systematic Review and Meta-Analyses) statement [13].

\section{Focus question}

The following question was developed according to the population, intervention, comparison, and outcome (PICO) study design (Table 1):

What are the clinical and radiographic treatment outcomes of the dental implants placed into the grafted sockets?

\section{Information sources}

The electronic databases MEDLINE (PubMed) was searched for relevant articles published until $1^{\text {st }}$ April 2019.

In MEDLINE (PubMed), the search was limited to humans, and English language. Also, a filter concerning "Article Type" was applied: controlled clinical trial, randomized controlled trial, evaluation studies, clinical study, clinical trial, comparative study, multicenter study, observational study, twin study, validation studies.

Table 1. The focus question development according to the PICO study design

\begin{tabular}{c|l}
\hline \multicolumn{1}{c|}{ Component } & \multicolumn{1}{c}{ Description } \\
\hline Population (P) & $\begin{array}{l}\text { Patients, older than 18 years and in good general health, requiring the placement of one or more implants in grafted } \\
\text { sockets }\end{array}$ \\
\hline Intervention (I) & Implant placement in grafted sockets \\
\hline Comparison (C) & Implant placement in non-grafted sockets \\
\hline Outcomes (O) & $\begin{array}{l}\text { Primary: implant survival. } \\
\text { Secondary: marginal-bone-level changes (mm), clinical parameters (i.e., bleeding on probing (\%), probing depth (mm), } \\
\text { occurrence of biological complications (i.e., peri-implant mucositis and/or peri-implantitis), aesthetic outcomes (pink } \\
\text { aesthetic score [PES], white aesthetic score [WES], PES/WES, Jemt's score) }\end{array}$ \\
\hline Focus question & What are the clinical and radiographic treatment outcomes of the dental implants placed into the grafted sockets? \\
\hline
\end{tabular}


In addition, a hand search was performed including reference lists of all full-text articles and the following scientific journals: "Clinical Oral Implants Research", "Clinical Implant Dentistry and Related Research", "European Journal of Oral Implantology", "Implant Dentistry", "International Journal of Oral \& Maxillofacial Implants", "International Journal of Periodontics and Restorative Dentistry", "Journal of Clinical Periodontology", "Journal of Oral Implantology", "International Journal of Oral and Maxillofacial Surgery", "Journal of Periodontology", "Journal of Prosthetic Dentistry", "Open Dentistry Journal", "Journal of Implants and Advanced Clinical Dentistry".

\section{Search}

The following search terms were used: (dental Implants [Mesh] OR implant) AND (bone regeneration [Mesh] OR socket preservation [Mesh] OR socket graft [Mesh] Or ridge preservation [Text word] OR augmentation [Text word]).

\section{Selection of studies}

During the first literature-selection stage, the titles and abstracts of all identified studies were screened for eligibility by two independent reviewers (AR and AC).

\section{Inclusion and exclusion criteria}

The following inclusion criteria were applied:

- Randomized controlled clinical trials, controlled clinical trials, and prospective studies with at least 12 months of follow-up with a minimum of 10 patients, older than 18 years old, having at least one implant inserted into the grafted socket;

- Grafting interventions performed prior to implant placement that aimed at preserving extraction sockets (i.e., procedures aimed at preserving the ridge volume within the envelope existing at the time of extraction [ $\underline{3}]$ ) were included;

- Studies with screw-type titanium implants that were placed into the healed sites (type IV implant placement [14]);

- Studies reporting on the specified primary or secondary treatment outcome.

At the second stage, the full texts of potentially eligible articles were reviewed and evaluated according to the following exclusion criteria:

- Animal studies;

- Retrospective studies, case reports, and crosssectional studies;

- Studies in which lateral ridge augmentation procedures and/or alveolar ridge contour augmentation and/or maxillary sinus floor elevation and/or augmentation of extraction [2] sockets were performed;

- Studies reporting on lateral and/or vertical bone augmentation procedures;

- Articles published in language other than English.

Differences between reviewers were resolved by discussion and consensus. The level of interexaminer agreement for the first- and second literature-selection stages was expressed by Cohen's kappa-score.

\section{Data extraction and data items}

From the selected articles fulfilling the inclusion criteria, the following data were retrieved into predefined tables:

- General information: study design, followup period, number of patients, patient-related information, including age, gender, smoking status, history of periodontitis (Table 2).

- Socket grafting procedures and implant related information: material used for socket grafting, Control group characteristics, use of systemic antibiotics, number of implants, time of implant placement, implant type, time of loading, maintenance program (Table 3).

- Treatment outcomes: implant survival, radiographic outcomes (i.e., marginal-bonelevel [MBL] changes), clinical parameters (i.e., bleeding on probing [BOP], probing depth (PD)), occurrence of biological complications (i.e., peri-implant mucositis and/or peri-implantitis), aesthetic outcomes (pink aesthetic score [PES], white aesthetic score [WES], PES/WES, Jemt's score), additional findings (Table 4).

\section{Quality assessment}

The Cochrane Collaboration's tool for assessing risk of bias was used in the case of controlled clinical trials [15] (Table 5). In six categories (random sequence generation, allocation concealment, blinding, incomplete outcome data, selective reporting, other potential risk of bias) a rating of low, unclear or high risk of bias was performed.

\section{Data synthesis}

A meta-analysis integrates the quantitative findings from separate but similar studies and provides a numerical estimate of the overall effect of interest. All meta-analyses were performed on randomized 
Table 2. Characteristics of the included studies

\begin{tabular}{|c|c|c|c|c|c|c|c|c|}
\hline Study & $\begin{array}{c}\text { Year of } \\
\text { publication }\end{array}$ & $\begin{array}{l}\text { Study } \\
\text { design }\end{array}$ & $\begin{array}{c}\text { Follow- } \\
\text { up period }\end{array}$ & $\begin{array}{c}\begin{array}{c}\text { No. of } \\
\text { patients }\end{array} \\
\end{array}$ & $\begin{array}{c}\text { Patient age } \\
\text { (years) }\end{array}$ & $\begin{array}{c}\text { Gender } \\
\text { (male/female) }\end{array}$ & Smoking status & History of periodontitis \\
\hline Tallarico et al. [27] & 2017 & $\mathrm{RCT}$ & 1.5 year & $\begin{array}{l}24 \text { patients. } \\
\text { Test: } 12 ; \\
\text { Control: } 12 \\
\end{array}$ & 53.9 (range 37 to 67 ) & $8 / 16$ & No smokers were included & Untreated periodontitis was an exclusion criteria \\
\hline Tallarico et al. [28] & 2016 & $\mathrm{RCT}$ & 1 year & $\begin{array}{l}24 \text { patients. } \\
\text { Test: } 12 ; \\
\text { Control: } 12\end{array}$ & $\begin{array}{c}\text { Test: } \\
56.2 \text { (range } 42 \text { to } 67) ; \\
\text { Control: } \\
51.6 \text { (range } 37 \text { to } 67)\end{array}$ & $8 / 16$ & No smokers were evaluated & Untreated periodontitis was an exclusion criteria \\
\hline Cardaropili et al. [29] & 2015 & $\mathrm{RCT}$ & 1 year & 41 & $47.2(\mathrm{SD} 12.9)$ & $24 / 17$ & NR & Periodontitis patients excluded \\
\hline Barone et al. [30] & 2012 & $\mathrm{RCT}$ & 3 years & $\begin{array}{l}40 \text { patients. } \\
\text { Test: } 20 \text {; } \\
\text { Control: } 20 \\
\end{array}$ & Range 26 to 69 & $16 / 24$ & $\begin{array}{c}\text { Smokers included. } \\
12 \text { participants }(30 \%) \\
\text { (6 in each group) were smokers }\end{array}$ & NR \\
\hline Marconcini et al. [31] & 2018 & $\mathrm{RCT}$ & 4 years & $\begin{array}{l}42 \text { patients. } \\
\text { Test } 1: 15 ; \\
\text { Test } 2: 14 ; \\
\text { Control: } 13\end{array}$ & $52.8(\mathrm{SD} 2.31)$ & $17 / 25$ & NR & NR \\
\hline Felice et al. [32] & 2015 & $\mathrm{RCT}$ & 1 year & $\begin{array}{l}50 \text { patients. } \\
\text { Test: } 25 ; \\
\text { Control: } 25\end{array}$ & $\begin{array}{c}\text { Test: } \\
\text { 53.08 (range } 39 \text { to } \\
72 \text { ); } \\
\text { Control: } \\
51.32 \text { (range } 32 \text { to } 71 \text { ) }\end{array}$ & $25 / 25$ & $\begin{array}{c}\text { Test: } \\
\text { non-smokers (16); } \\
\text { up to } 10 \text { cigarettes/day (9); } \\
\text { more than } 10 \text { cigarettes/day }(0) \\
\text { Control: } \\
\text { non-smokers }(14) ; \\
\text { up to } 10 \text { cigarettes/day (8); } \\
\text { more than } 10 \text { cigarettes/day (3) }\end{array}$ & Untreated periodontitis was an exclusion criteria \\
\hline Esposito et al. [33] & 2015 & $\mathrm{RCT}$ & 1 year & $\begin{array}{l}106 \text { patients. } \\
\text { Test: } 52 ; \\
\text { Control: } 54\end{array}$ & $\begin{array}{c}\text { Test: } \\
50 \text { (range } 30 \text { to } 72) ; \\
\text { Control: } \\
48 \text { (range } 28 \text { to } 70)\end{array}$ & $46 / 60$ & $\begin{array}{l}\text { Three groups: } \\
\text { non-smokers; } \\
\text { up to } 10 \text { cigarettes/day; } \\
\text { more } 10 \text { cigarettes/day }\end{array}$ & Untreated periodontitis was an exclusion criteria \\
\hline Esposito et al. [34] & 2017 & $\mathrm{RCT}$ & 1 year & $\begin{array}{l}210 \text { patients. } \\
\text { Test: } 70 ; \\
\text { Control 1: } 70 ; \\
\text { Control 2: } 70\end{array}$ & $\begin{array}{c}\text { Test: } \\
\text { 55.8 (SD 11.6); } \\
\text { Control 1: } \\
55.3 \text { (SD 11); } \\
\text { Control 2: } \\
\text { 53.5 (SD 13.4) }\end{array}$ & $\begin{array}{c}\text { Test: } \\
\text { 37/33 } \\
\text { Control 1: } \\
\text { 34/36 } \\
\text { Control 2: } \\
\text { 36/34 }\end{array}$ & $\begin{array}{l}\text { Three groups: } \\
\text { non-smokers; } \\
\text { up to } 10 \text { cigarettes/day; } \\
\text { more } 10 \text { cigarettes/day }\end{array}$ & Untreated periodontitis was an exclusion criteria \\
\hline
\end{tabular}

$\mathrm{RCT}=$ randomized clinical trial $; \mathrm{NR}=$ not reported $\mathrm{SD}=$ standard deviation

All papers included systemically healthy patients. 
Table 3. Socket preservation procedures and implant characteristics

\begin{tabular}{|c|c|c|c|c|c|c|c|c|}
\hline Study & Socket grafting materials & Control group & Systemic antibiotics & $\begin{array}{c}\text { No. of } \\
\text { implants }\end{array}$ & $\begin{array}{c}\text { Time of implant } \\
\text { placement }\end{array}$ & Implant type & Loading protocol & $\begin{array}{l}\text { Maintenance } \\
\text { program }\end{array}$ \\
\hline $\begin{array}{c}\text { Tallarico et al. } \\
\text { [27] }\end{array}$ & $\begin{array}{c}\text { Corticocancellous porcine } \\
\text { bone }\left(\mathrm{GENOSS}, \text { OsteoBiol }^{\mathbb{}}\right)+ \\
\text { portice derma }\left(\mathrm{OsteoBiol}^{\mathbb{}}\right)\end{array}$ & 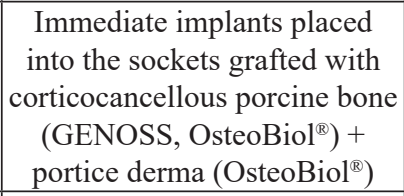 & $\begin{array}{c}\text { Prophylactic } \\
\text { preoperatively; } \\
4 \text { days postoperatively }\end{array}$ & \begin{tabular}{|c|}
24 implants. \\
Test: $12 ;$ \\
Control: 12
\end{tabular} & 4 months & Osstem $^{\circledR} 7 \mathrm{~mm}$ wide & $\begin{array}{l}4 \text { months: definitive } \\
\text { restorations }\end{array}$ & 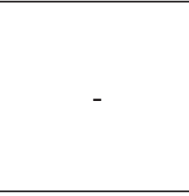 \\
\hline $\begin{array}{c}\text { Tallarico et al. } \\
{[28]}\end{array}$ & $\begin{array}{c}\text { Corticocancellous porcine } \\
\text { bone }+ \text { extracellular resorbable } \\
\text { membrane. } \\
\text { GENOSS, OsteoBiol }{ }^{\circledR}+\text { portice } \\
\text { derma }\left(\text { OsteoBiol }{ }^{\mathbb{Q}}\right)\end{array}$ & \begin{tabular}{|c|} 
Immediate implants placed \\
into the sockets grafted with \\
corticocancellous porcine bone \\
$\left(\right.$ GENOSS, OsteoBiol $\left.^{\mathbb{}}\right)+$ \\
portice derma $\left(\right.$ OsteoBiol $\left.^{\mathbb{}}\right)$ \\
\end{tabular} & \begin{tabular}{|c|} 
Prophylactic \\
preoperatively; \\
4 days postoperatively
\end{tabular} & \begin{tabular}{|c|}
24 implants. \\
Test: $12 ;$ \\
Control: 12
\end{tabular} & 4 months & Osstem $^{\circledR} 7 \mathrm{~mm}$ wide & $\begin{array}{l}4 \text { months: definitive } \\
\text { restorations }\end{array}$ & $\begin{array}{l}\text { Recall visits } \\
\text { every } 6 \\
\text { months, up to } \\
1 \text { year. }\end{array}$ \\
\hline $\begin{array}{c}\text { Cardaropoli et al. } \\
{[29]}\end{array}$ & \begin{tabular}{|c|} 
Deproteinized bovine bone \\
graft $\left(\right.$ Geistlich Bio-Oss $\left.{ }^{\circledR}\right)+$ \\
absorbable collagen membrane \\
$\left(\right.$ Geistlich Bio-Gide $\left.{ }^{\circledR}\right)$
\end{tabular} & $\begin{array}{c}\text { Implants placed in naturally } \\
\text { healed sites }\end{array}$ & No & $\begin{array}{l}48 \text { implants. } \\
\text { Test: } 24 ; \\
\text { Control: } 24\end{array}$ & 4 months & $\begin{array}{l}4 \text { to } 5 \mathrm{~mm} \text { diameter, } \\
\text { conical shape. } \\
\text { (Biomet } / 3 \mathrm{i} \text { ) }\end{array}$ & 4 months & NR \\
\hline Barone et al. [30] & $\begin{array}{c}\text { Corticocancellous porcine } \\
\text { bone particles }\left(\text { OsteoBiol }^{\circledR}\right) \\
+ \text { collagene membrane } \\
(\text { OsteoBiol }\end{array}$ & $\begin{array}{c}\text { Implants placed in naturally } \\
\text { healed sites }\end{array}$ & $\begin{array}{c}\text { Prophylactic } \\
\text { preoperatively; } \\
4 \text { days postoperatively }\end{array}$ & \begin{tabular}{|c|}
40 implants. \\
Test: 20 \\
Control: 20
\end{tabular} & 4 months & $\begin{array}{l}3.3 \text { to } 5 \mathrm{~mm} \text { diameter, } \\
10 \text { to } 13 \mathrm{~mm} \text { length } \\
\left.\text { (Sweden \& Martina }{ }^{\circledR}\right)\end{array}$ & 4 months & $\begin{array}{l}\text { Oral hygiene } \\
\text { every } 3 \\
\text { months }\end{array}$ \\
\hline $\begin{array}{c}\text { Marconcini et al. } \\
{[31]}\end{array}$ & 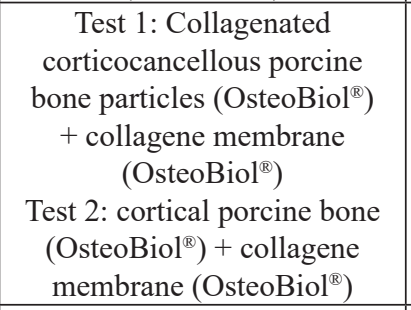 & $\begin{array}{c}\text { Implants placed in naturally } \\
\text { healed sites }\end{array}$ & $\begin{array}{c}\text { Prophylactic } \\
\text { preoperatively; } \\
4 \text { days postoperatively }\end{array}$ & \begin{tabular}{|l|}
42 implants. \\
Test $1: 15 ;$ \\
Test $2: 14 ;$ \\
Control: 13
\end{tabular} & 3 months & $\begin{array}{l}\text { Diameter: NR; } \\
\text { BT Evo; Biotech }\end{array}$ & $\begin{array}{l}\text { Two-stage surgery } \\
\quad 4 \text { month }\end{array}$ & $\begin{array}{l}\text { Personalized } \\
\text { maintenance } \\
\text { programme } \\
\text { and recall } \\
\text { visits }\end{array}$ \\
\hline Felice et al. [32] & $\begin{array}{l}\text { Algae-derived frios algipore } \\
\left(\text { Dentsply Friadent }{ }^{\circledR}\right)+ \\
\text { Geistlich Bio-Gide }^{\circledR}\end{array}$ & $\begin{array}{c}\text { Immediate implant placement } \\
\text { bone-to-implant gaps filled } \\
\text { with algae-derived bone } \\
\text { substitute } \\
\end{array}$ & \begin{tabular}{|c|} 
Prophylactic \\
preoperatively; \\
4 days postoperatively
\end{tabular} & \begin{tabular}{|l}
50 implants. \\
Test: $25 ;$ \\
Control: 25
\end{tabular} & 4 months & $\begin{array}{c}\text { Diameter: } 3.8 / 4.5 / 5.5 \\
\text { mm, length: } \\
\text { 8/9.5/11/13/15 mm } \\
\text { Xive S plus, Dentsply } \\
\end{array}$ & \begin{tabular}{|c|} 
Immediate restoration \\
$(>35 \mathrm{~N})$ \\
4 months $(<35 \mathrm{~N})$ \\
4 months definitive crown \\
\end{tabular} & $\begin{array}{c}\text { Oral hygiene } \\
\text { and recall } \\
\text { visit every } 6 \\
\text { months } \\
\end{array}$ \\
\hline $\begin{array}{c}\text { Esposito et al. } \\
\text { [33] }\end{array}$ & $\begin{array}{c}\text { Deproteinized bovine bone } \\
\text { graft }\left(\text { Bio-Oss }{ }^{\mathbb{Q}}\right)+\text { absorbable } \\
\text { collagen membrane }(\text { Geistlich } \\
\left.\text { Bio-Gide }^{\mathbb{B}}\right) \\
\end{array}$ & $\begin{array}{c}\text { Immediate implant placement } \\
35 \text { implants }(40 \%) \text { bone-to- } \\
\text { implant gaps were filled with } \\
\text { anorganic bovine bone } \\
\end{array}$ & \begin{tabular}{|c|} 
Prophylactic \\
preoperatively; \\
4 days postoperatively
\end{tabular} & \begin{tabular}{|c}
106 implants. \\
Test: $54 ;$ \\
Control: 52
\end{tabular} & 4 months & $\begin{array}{c}\text { Diameter: } 4 / 5 \mathrm{~mm} \\
\text { Length: } \\
\text { 7/8.5/10/11.5/13/15 mm } \\
\left(\text { MegaGen }^{\circledR}\right) \\
\end{array}$ & $\begin{array}{c}\text { Immediate restoration } \\
(>35 \mathrm{~N}) ; \\
4 \text { months }(<35 \mathrm{~N}) ; \\
4 \text { months definitive crown } \\
\end{array}$ & NR \\
\hline $\begin{array}{c}\text { Esposito et al. } \\
{[34]}\end{array}$ & $\begin{array}{c}\text { Collagenated corticocancellous } \\
\text { porcine bone particles } \\
\left(\text { OsteoBiol }{ }^{\circledR}\right)+\text { resorbable } \\
\text { membrane derived from equine } \\
\text { pericardium }\left(\text { OsteoBiol }{ }^{\circledR}\right) \\
\end{array}$ & $\begin{array}{c}\begin{array}{c}\text { Control 1: immediate placed } \\
\text { implants. } \\
\text { Control 2: immediate-delayed } \\
\text { placed implants after } 6 \text { weeks } \\
\text { post extraction }\end{array} \\
\end{array}$ & $\begin{array}{c}\text { Prophylactic } \\
\text { preoperatively; } \\
4 \text { days postoperatively }\end{array}$ & $\begin{array}{l}210 \text { implants. } \\
\text { Test: } 70 ; \\
\text { Control 1: } 70 \\
\text { Control 2: } 70\end{array} \mid$ & 4 months & $\begin{array}{l}\text { Diameter: } 3.5 / 4.3 / 5 \\
\text { mm; length: } \\
8.5 / 10 / 11.5 / 13 / 15 \mathrm{~mm} \\
{\left(\text { Nobel Biocare }^{\circledR}\right)}^{\text {(Nobel }}\end{array}$ & $\begin{array}{c}\text { Two-stage surgery; } \\
\text { Loading with prov. } \\
\text { Crowns } 4 / 6 \text { months after } \\
\text { surgery }\end{array}$ & $\begin{array}{l}\text { Oral hygiene } \\
\text { and recall } \\
\text { visit every } 6 \\
\text { months }\end{array}$ \\
\hline
\end{tabular}

aProphylactic preoperatively $2 \mathrm{~g}$ amoxicillin or $600 \mathrm{mg}$ clindamycin (in case of allergy); 4 days postoperatively $1 \mathrm{~g}$ amoxicillin or $300 \mathrm{mg}$ clindamycin twice a day.

Table 4. Treatment outcomes

\begin{tabular}{|c|c|c|c|c|c|c|}
\hline Study & $\mid \begin{array}{c}\text { Implant survival } \\
(\%)\end{array}$ & $\begin{array}{l}\text { Radiographic bone } \\
\text { assessment }\end{array}$ & $\begin{array}{c}\text { Clinical } \\
\text { parameters }^{\mathrm{a}}\end{array}$ & $\begin{array}{c}\text { Peri-implant } \\
\text { diseases }\end{array}$ & Aesthetic outcomes $^{b}$ & Other findings \\
\hline Tallarico et al. [27] & $\begin{array}{c}\text { Test: } 100 \% ; \\
\text { Control: } 100 \%\end{array}$ & \begin{tabular}{|c|} 
MBL changes. \\
Test: $0.23(\mathrm{SD} 0.06) \mathrm{mm} ;$ \\
Control: $0.63(\mathrm{SD} 0.31) \mathrm{mm} ; \mathrm{P}=0.001$ \\
\end{tabular} & NR & NR & $\begin{array}{c}\text { PES score. } \\
\text { Test group: 12.2 (SD 1.2); } \\
\text { Control group: } 10.6(\mathrm{SD} 1.8) ; \mathrm{P}=0.019\end{array}$ & - \\
\hline Tallarico et al. [28] & $\begin{array}{c}\text { Test: } 100 \% ; \\
\text { Control: } 100 \%\end{array}$ & $\begin{array}{c}\text { MBL changes. } \\
\text { Test: } 0.23(\mathrm{SD} 0.06) \mathrm{mm} ; \\
\text { Control: } 0.63(\mathrm{SD} 0.31) \mathrm{mm} ; \mathrm{P}=0.01\end{array}$ & NR & NR & $\begin{array}{c}\text { PES score. } \\
\text { Test group: } 11.7(\text { SD 1.2); } \\
\text { Control group: } 10.7(\text { SD 1.5); } \mathrm{P}=0.081\end{array}$ & - \\
\hline Cardaropoli et al. [29] & $\begin{array}{c}\text { Test: } 100 \% \\
\text { Control: } 100 \%\end{array}$ & $\begin{array}{c}\text { MBL changes. } \\
\text { Test: } 0.33(\mathrm{SD} 0.28) \mathrm{mm} \\
\text { Control: } 0.35(\mathrm{SD} 0.27) \mathrm{mm} ; \mathrm{P}=0.8\end{array}$ & NR & NR & 然 & $\begin{array}{l}\text { During implant placement additional bone } \\
\text { grafting was necessary in } 14 \text { implants in Test } \\
\text { group }(58.33 \%), \mathrm{P}<0.05 . \\
\text { MBL }>1 \text { mm during the first year: } \\
\text { Test: } 1 \text { implant; } \\
\text { Control: } 2 \text { implants }(\mathrm{P}=0.98)\end{array}$ \\
\hline Barone et al. [30] & $\begin{array}{c}\text { Test: } 95 \% \text {; } \\
\text { Control: } 95 \%\end{array}$ & $\begin{array}{c}\text { MBL changes. } \\
1 \text { year: } \\
\text { Test: } 0.75 \text { (SD } 0.3) \mathrm{mm} ; \\
\text { Control: } 0.76 \text { (SD } 0.3) \mathrm{mm} ; \mathrm{P}=0.82 \\
2 \text { years: } \\
\text { Test: } 0.83 \text { (SD } 0.2) \mathrm{mm} ; \\
\text { Control: } 0.84 \text { (SD } 0.2) \mathrm{mm} ; \mathrm{P}=0.66 \\
3 \text { years: } \\
\text { Test: } 1 \text { (SD } 0.2) \mathrm{mm} ; \\
\text { Control: } 1.02(\mathrm{SD} 0.3) \mathrm{mm} ; \mathrm{P}=0.52\end{array}$ & NR & NR & NR & $\begin{array}{l}\text { During implant placement additional bone } \\
\text { grafting was necessary in } 13 \text { implants in the Test } \\
\text { group and } 10 \text { in the Control group }(\mathrm{P}=0.02)\end{array}$ \\
\hline Marconcini et al. [31] & $\begin{array}{l}\text { Test 1: } 100 \% \text {; } \\
\text { Test 2: } 100 \% \text {; } \\
\text { Control: } 100 \%\end{array}$ & $\begin{array}{c}\text { MBL changes. } \\
\text { Test } 1: 1.14(\text { SD } 0.23) \mathrm{mm} \\
\text { Test } 2: 1.13(\text { SD } 0.29) \mathrm{mm} \\
\text { Control: } 1.95(\text { SD } 0.07) \mathrm{mm} \text {. } \\
\text { Significantly higher in control group } \\
\quad(\mathrm{P}<0.001)\end{array}$ & NR & NR & $\begin{array}{c}\text { PES score. } \\
\text { Test 1: } 9.42(\text { SD 0.75); } \\
\text { Test 2: } 8.53 \text { (SD 1.18); } \\
\text { Control: } 6.07 \text { (SD 1.89). } \\
\text { Significantly higher in Test 1 compared to } \\
\text { Test 2 and Control groups }(\mathrm{P}=0.02)\end{array}$ & $\begin{array}{l}\text { During implant placement additional bone } \\
\text { grafting was necessary in } 6 \text { implants in the } \\
\text { Control group }\end{array}$ \\
\hline Felice et al. [32] & $\begin{array}{l}\text { Test: } 100 \% \text {; } \\
\text { Control: } 92 \%\end{array}$ & $\begin{array}{c}\text { MBL changes. } \\
\text { Test } 0.19(\mathrm{SD} \mathrm{0.1}) \mathrm{mm} \\
\text { Control } 0.13(\mathrm{SD} 0.09) \mathrm{mm} ; \mathrm{P}<0.001\end{array}$ & NR & NR & $\begin{array}{c}\text { PES score. } \\
\text { Test group: } 12.22 ; \\
\text { Control group: } 12.78 ; \mathrm{P}=0.09\end{array}$ & - \\
\hline Esposito et al. [33] & $\begin{array}{l}\text { Test: } 100 \% \text {; } \\
\text { Control: } 96 \%\end{array}$ & $\begin{array}{c}\text { MBL changes. } \\
\text { Test: } 0.27(\text { SD } 0.14) \mathrm{mm} \\
\text { Control: } 0.13(\text { SD } 0.16) \mathrm{mm} . \\
\text { Significantly higher for the test } \\
\text { implants }(\mathrm{P}<0.036)\end{array}$ & NR & NR & $\begin{array}{c}\text { PES score. } \\
\text { Test group 12.8 (SD 1.4); } \\
\text { Control group: } 13(\mathrm{SD} 1.5) ; \mathrm{P}=0.615\end{array}$ & - \\
\hline Esposito et al. [34] & $\begin{array}{l}\text { Test: } 98 \% \text {; } \\
\text { Control 1: } 94 \% \text {; } \\
\text { Control 2: } 94 \%\end{array}$ & $\begin{array}{c}\text { MBL loss. } \\
\text { Test: } 0.31(\text { SD } 0.16) \mathrm{mm} \text {; } \\
\text { Control 1: } 0.25(\text { SD } 0.17) \mathrm{mm} \text {; } \\
\text { Control 2: } 0.29(\text { SD } 0.14) \mathrm{mm} \text {. } \\
\text { Significantly higher for the test } \\
\text { implants }(\mathrm{P}<0.0001)\end{array}$ & NR & NR & $\begin{array}{c}\text { PES score. } \\
\text { Test group: 11.78 (SD 1.1); } \\
\text { Control 1: } 12.52(\text { SD 1.08); } \\
\text { Control 2: } 12.49 \text { (SD 0.96); P }<0.0001\end{array}$ & - \\
\hline
\end{tabular}

${ }^{\mathrm{a}}$ Clinical parameters: probing depth, bleeding on probing, mucosal recession.

bAesthetic outcomes: papilla index, PES (pink aesthetic score).

$\mathrm{MBL}=$ marginal-bone-level; $\mathrm{PES}=$ pink aesthetic score (mean value); $\mathrm{SD}=$ standard deviation; $\mathrm{NR}=$ not reported. 
Table 5. Assessment of the risk of bias for included controlled clinical studies

\begin{tabular}{l|c|c|c|c|c|c|c}
\hline \multicolumn{1}{c|}{ Study } & $\begin{array}{c}\text { Random } \\
\text { sequence } \\
\text { generation }\end{array}$ & $\begin{array}{c}\text { Allocation } \\
\text { concealment }\end{array}$ & Blinding & $\begin{array}{c}\text { Incomplete } \\
\text { outcome } \\
\text { data }\end{array}$ & $\begin{array}{c}\text { Selective } \\
\text { reporting }\end{array}$ & $\begin{array}{c}\text { Other } \\
\text { bias }\end{array}$ & $\begin{array}{c}\text { Summary } \\
\text { assessment }\end{array}$ \\
\hline Tallarico et al. [27] & Low & Low & Low & Low & Low & Low & Low \\
\hline Cardaropoli et al. [29] & Low & Unclear & Unclear & Low & Low & Low & Unclear \\
\hline Barone et al. [30] & Unclear & Low & Low & Low & Low & Low & Low \\
\hline Marconcini et al. [31] & Low & Low & Unclear & Low & Low & Unclear & Low \\
\hline Felice et al. [32] & Low & Unclear & Unclear & Low & Low & High & Unclear \\
\hline Esposito et al. [33] & Low & Low & Low & Low & Low & Unclear & Low \\
\hline Esposito et al. [34] & Low & High & Low & Low & Low & Unclear & Low \\
\hline
\end{tabular}

controlled clinical trials that reported the clinical outcomes of non-surgical periodontitis treatment utilizing different adjunctive antiseptics.

Thus, each study provided estimates of outcome measures. The goal was to obtain global estimates of these measures and to test whether they differed significantly. Global estimates of a proportion can be obtained by simply pooling together the data from each study. However, a test for significance cannot be applied to such pooled data, as these studies are heterogeneous with respect to study population and treatment protocol. Therefore, individual trials were pooled, and the weighted mean differences for the MBL changes, together with their $95 \%$ confidence intervals (CIs), were calculated. Under the fixedeffects model, it is assumed that all studies come from a general population and that the size is not significantly different among the different trials. This assumption was tested by the heterogeneity test using the Cochran Q statistics. We considered that in our case the random-effects model (the Der Simonian and Liard method) [16] was more appropriate to use since it took into account both the random variation within the studies and the variation among different studies. Later findings indicated the fixed-effects model might be invalid. Indeed, the random-effects model tended to give a more conservative estimate (i.e, with a wider $\mathrm{CI})$, but the results from the 2 models usually agreed well.

\section{RESULTS}

\section{Search results}

The initial electronic search resulted in the identification of 115 titles. Following the evaluation of titles and abstracts, 99 publications were excluded $($ Cohens kappa $=0.927)$. The remaining 16 full-text articles were evaluated. The reasons for exclusion were as follows: (i) studies included $<10$ patients in the Test group [17-19], did not assess the outcomes of dental implants $[20,21]$, follow-up period $<1$ year
[22-26]. Finally, 8 articles were included into the analysis (Figure 1).

\section{Characteristics of the included studies}

The included studies are described in Tables 1 to 3. Two of the studies involved the same patient sample that was evaluated at different followup periods $[27,28]$, therefore, a total of 7 original clinical investigations were included. All of them were designed as randomized controlled trials with a follow-up period ranging from 1 to 4 years. Control group participants in 3 of the studies had implants placed into naturally healed sites [29-31]. In the remaining 4 investigations Control group participants were treated with immediately placed implants $[27,32-34]$ or were inserted 6 weeks post extraction [34]. Additionally, in the latter 4 investigations, immediate implants were inserted either into grafted sockets [27] or the gap between the implant and the bone was filled with a bone-fill material [32-34].

This systematic review pooled data collected from 512 patients (243 men, 270 women). All investigations included systemically healthy patients with a mean age range of 42.7 to 55.8 years. Smokers were included in 4 of the studies [30,32-34]. Smoking habit was an exclusion criteria in one investigation [27] and patient smoking status was not reported in two of the studies $[29,31]$. In 5 of the 7 studies, untreated periodontitis $[27,32-34]$ or a history of periodontitis [29] was an exclusion criteria. Patients' periodontal status was not reported in two studies $[\underline{30}, \underline{31}]$ (Table 2).

Socket grafting in all seven studies involved guided bone regeneration concept with the use of bone substitute material covered with a barrier membrane (Table 3). As a bone substitute, either xenogenous (six studies) or alloplastic (one study) bone-filler particles were used. As a barrier membrane, either collagen membranes (in five studies), porcine derma (one study) or a pericardium membrane (one study) 


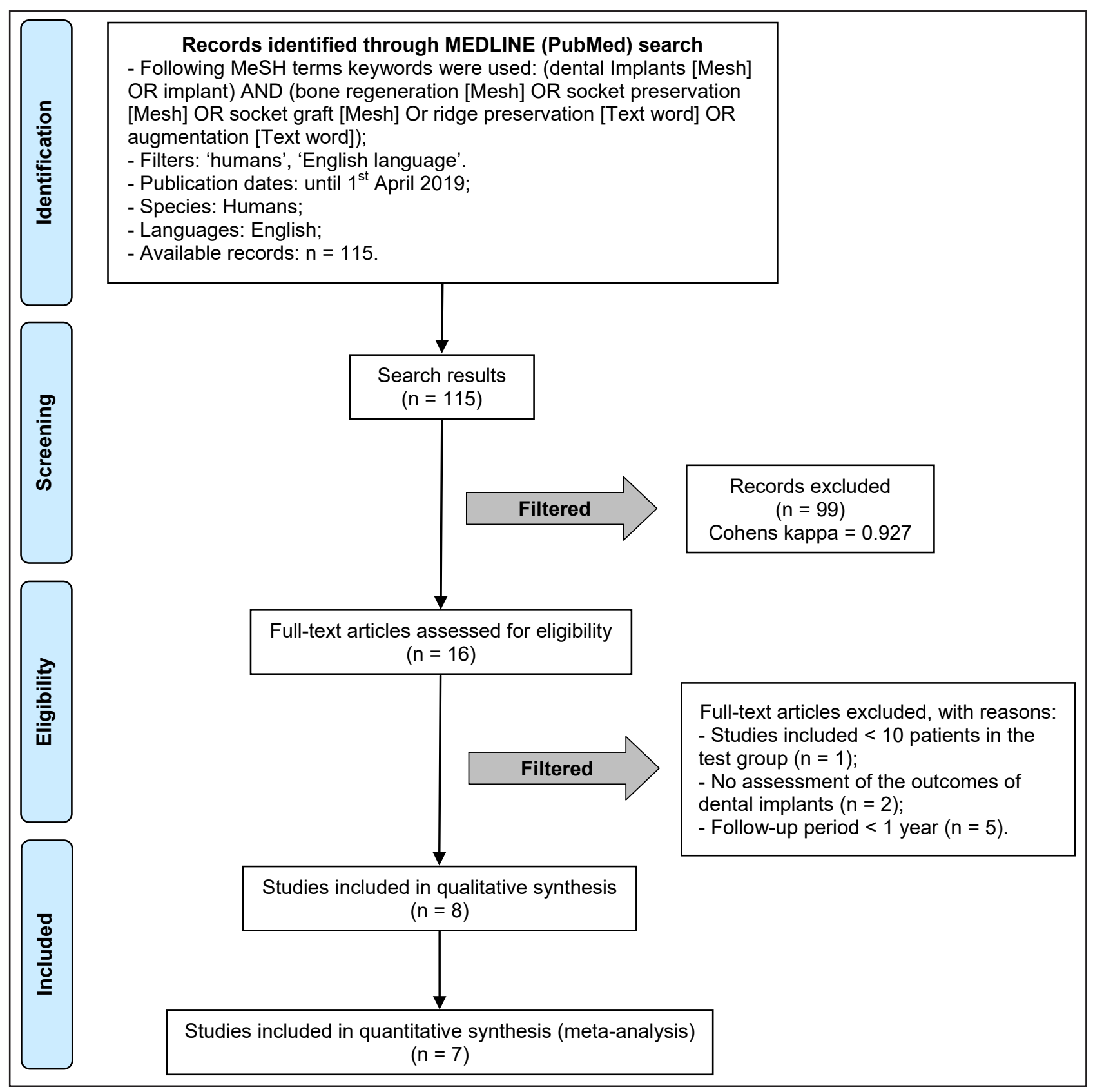

Figure 1. PRISMA 2009 flow diagram.

were used. In all but one study [29], all patients received prophylactic antibiotics preoperatively and continuously 4 to 6 days postoperatively (Table 4).

Of the total of 520 implants included, 232 were inserted into the previously grafted sockets (3 to 7 months following the grafting) (test), fiftyseven were inserted into the naturally healed sites, and 231 were placed immediately (or 6 week post extraction) after tooth extraction (controls). The diameter of the implants ranged from 3.3 to $5.5 \mathrm{~mm}$, and one investigation included only implants with a diameter of $7 \mathrm{~mm}$ [27]. In 5 of the studies, patients were enrolled into either a personalized or regular maintenance program with 3- to 6-month intervals $[\underline{27}, \underline{30}, \underline{32}, \underline{34}]$.
The assessment of risk of bias in the included randomized trials is presented in Table 5. A low risk of bias was noted in 5 studies according to 4 (3 studies), 5 (2 studies) or all 6 domains (1 study) $[27,30,31,33,34]$. The remaining 2 studies were judged to have an unclear risk of bias based on 2 ('allocation concealment' and 'blinding') domains $[\underline{29}, \underline{32}]$.

\section{The reported clinical outcomes of the implants}

Implant survival, which was defined as a primary outcome, ranged from 95 to $100 \%$ for the Test group implants and from 92 to $100 \%$ for the implants in the Control groups (Table 4). 
The mean MBL changes were reposted in all 7 investigations included. Two of them revealed no significant difference between the implants placed into the naturally healed sites or the previously grafted sockets $[\underline{29}, \underline{30}]$. In contrast, a single study reported higher MBL loss for the implants placed into the naturally healed sites versus grafted sockets [31]. In five investigations where immediate implants were the Control group, three of them found significantly higher MBL resorption for the implants placed into previously grafted sockets, compared to the immediately inserted ones [32-34]. Contradictory findings were reported by Tallarico et al. [27], where immediately placed implants experienced significantly higher rates of the MBL loss.

A pink aesthetic score (PES) was reported in five of the included studies [27,31-34]. In two of them, no difference was noted between the implants placed into the grafted sockets or immediately placed ones $[32,33]$, whereas one study reported significantly higher PES scores for the immediately inserted implants [34]. Conversely, two investigations found superior soft tissue measurements for the implants placed into the healed, previously grafted sockets, compared to the immediately placed ones [27] and implants placed into the naturally healed sites [31]. Additionally, it has to be pointed out that none of the included studies reported the clinical parameters (i.e., BOP, PD) or the occurrence of peri-implant diseases (i.e., peri-implant mucositis or peri-implantitis). Moreover, three of the investigations revealed that additional bone grafting procedures were more often necessary around the implants placed into the naturally healed sites, compared to the previously grafted post extraction sockets [29-31]. Two of these studies found this difference to be statistically significant $[\underline{29}, \underline{30}]$.

\section{Meta-analysis}

A meta-analysis was conducted to assess MBL changes in the following groups:

- Implants placed in the grafted sockets $(n=73$ implants) vs. implants inserted into the nongrafted bone ( $\mathrm{n}=57$ implants).

- Implants placed in the grafted sockets (159 implants) vs. immediately placed implants (231 implants).

The results showed significantly higher marginal bone loss for the implants in previously non-grafted healed sites than for the ones placed in the grafted sockets (weighted mean difference $(\mathrm{WMD})=-1.961,95 \%$ confidence interval [CI] [-3.793 to -0.13]; degrees of freedom $[\mathrm{df}]=3$; heterogeneity test $[\mathrm{Q}]=54.36$;
$\mathrm{P}<0.0001$ ) (Figure 2).

The comparison between implants in the grafted sockets vs. immediate implant placement (or 6 weeks post extraction) demonstrated no significant difference between the two implant groups (WMD $=0.194,95 \%$ CI [-0.342 to 0.731]; $\mathrm{df}=4 ; \mathrm{Q}=28.29 ; \mathrm{P}<0.0001)$ (Figure 3).

\section{DISCUSSION}

The aim of the present systematic review was to investigate the clinical performance of the implants placed in the previously grafted sockets. The included clinical studies presented methodological differences in terms of the variables assessed to measure the outcomes, and the Control groups (immediate implant placement vs. implants in the healed non-grafted sites).

Based on the included 7 randomized clinical trials, the implants' survival rates ranged between $95 \%$ and $100 \%$ after 1 to 4 years of implant placement, with no significant differences between implants placed into previously augmented sockets and those inserted into the spontaneously healed sites. These findings corroborate the survival rates noted for the implants placed into the laterally augmented alveolar ridge sites $(78.2$ to $100 \%)$ [35] and for implants inserted into the laterally augmented sinuses ( 88.6 to $100 \%)$ []]. Furthermore, the implant-survival rates following socket grafting were within the range of those previously reported for implants placed in pristine sites (93.2 to $100 \%$ ) [7] .

The present meta-analysis points toward significantly higher MBL loss for the implants in previously nongrafted healed sites than for implants placed in grafted sockets. Furthermore, the 3 studies included in the meta-analysis revealed that additional bonegrafting procedures were often required during implant placement at the sites without previous socket grafting [29-31]. These findings are comparable with the results of the previous metaanalysis, in which implant placement without bone augmentation resulted in significantly higher radiographic marginal bone loss compared to at the sites where dehiscence-type defects were augmented using a xenograft bone substitute and a collagen membrane [36]. However, out of three studies included in the present quantitative analysis, significant differences in terms on the MBL changes were reported in the investigation with the longest follow-up period (4 years [31] versus 1 [29] and 3 years [이]). Therefore, the follow-up period might be a critical factor in the treatment outcomes. 


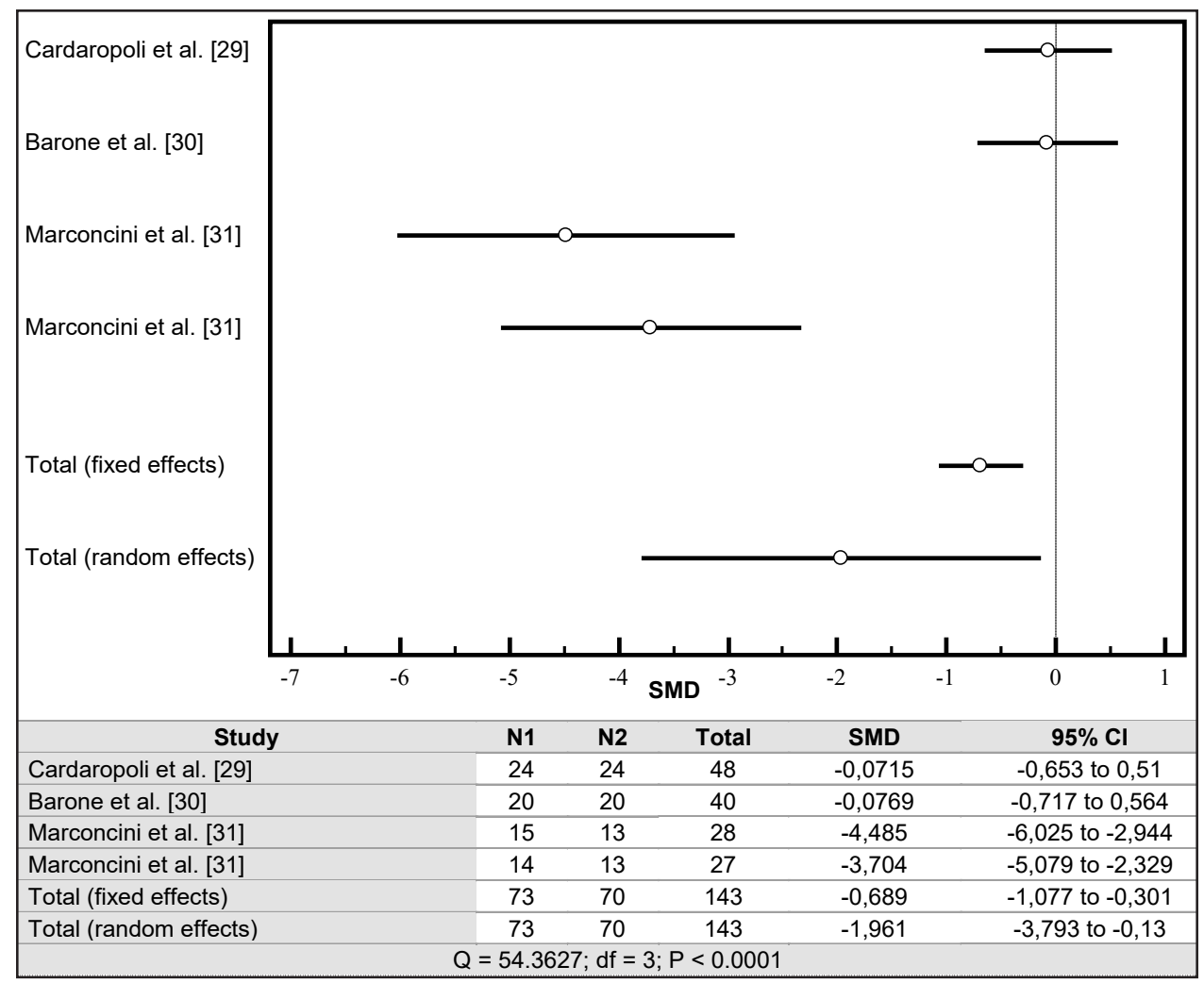

Figure 2. Forest plot of odds ratio $(95 \% \mathrm{CI})$ for marginal bone level changes between the implants placed in the grafted sockets and implants inserted in previously non-grafted sites. $\mathrm{CI}=$ confidence interval; $\mathrm{SMD}=$ standardized mean difference; $\mathrm{N} 1=$ implants placed in the grafted sockets; N2 = implants inserted in previously non-grafted sites; $\mathrm{df}=$ degrees of freedom; $\mathrm{Q}=$ heterogeneity test.

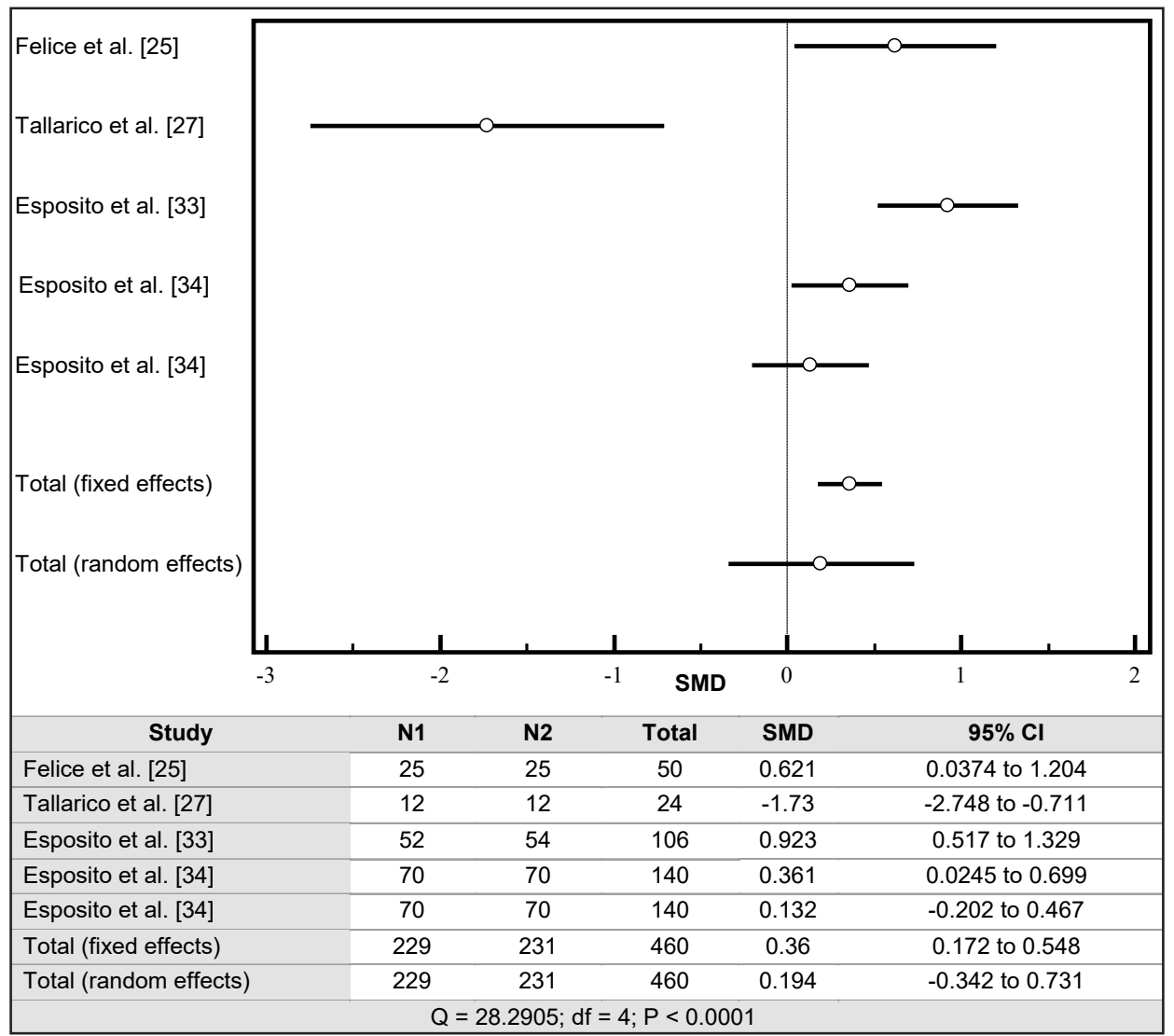

Figure 3. Forest plot of odds ratio $(95 \% \mathrm{CI})$ for marginal-bone-level changes between the implants placed in grafted sockets and immediate implant placement. $\mathrm{CI}=$ confidence interval; $\mathrm{SMD}=$ standardized mean difference; $\mathrm{N} 1=$ implants placed in grafted sockets; $\mathrm{N} 2=$ immediate implant placement; $\mathrm{df}=$ degrees of freedom; $\mathrm{Q}=$ heterogeneity test. 
Further comparison of the MBL changes between implants in the grafted sockets vs. immediate implant placement demonstrated no significant difference between the two approaches. Similar results were obtained in a previous systematic review, in which implant placement into fresh extraction sockets versus delayed implants into healed sockets resulted in comparable MBL changes [37]. However, among the 4 investigations included in the present metaanalysis, an inconsistency regarding the treatment outcomes was noted. Specifically, while Tallarico et al. [27] found significantly higher MBL loss for the immediately inserted implants over those placed into the healed and previously grafted sockets, the remaining investigations reported significantly higher MBL for the implants in the latter group (i.e., with delayed implants in the previously grafted sockets) [32-34].

The soft-tissue changes around the implants were assessed by PES score in 5 of the included studies [27,31-34]. Again, inconsistency existed among the results of the included studies. In particular, while 2 studies found superior PES scores for the implants placed into the healed, previously grafted sockets [27] and implants placed into the naturally healed sites [31] as compared to the immediately placed ones, Esposito et al. [34] contrarily presented significantly better soft-tissue outcomes for the immediately inserted implants. The latter study corroborates the results of the previous 3-year clinical investigations, which found superior soft-tissue outcomes (PES scores) for the immediately placed and immediately restored implants, as compared to the conventionally restored implants installed in to the healed sites [38]. The authors noticed that superior aesthetic outcomes could be expected in young patients ( $\leq 30$ years) with implants in central incisor/cuspid areas and in the presence of bone recontouring [38].

Contradictory findings exist regarding the timing of implant placement and soft-tissue treatment outcomes.
A recent prospective clinical investigation reported no significant difference in PES values between the immediate and conventional implant placement after 1- and 8 years of follow-up [39]. This observation is supported by the 2 included studies in the present analysis that revealed comparable soft-tissue outcomes for the immediate and delayed implant placement $[32,33]$.

Recently, a meta-analysis has found the comparable prevalence of peri-implant mucositis and periimplantitis as well as implant loss for dental implants placed in either native or augmented sites (including vertical and/or horizontal ridge augmentation) at both the patient and implant levels [40]. In the present analysis, however, none of the studies reported the clinical parameters or the occurrence of biological implant complications, which prevents comparison with the previous investigations.

\section{CONCLUSIONS}

In terms of survival rates, placing dental implants in previously grafted sockets is a predictable treatment option. Differences could be found for the periimplant marginal-bone-level changes when compared with the implants placed into the non-grafted sites. Other objective quantitative methods must be considered in future studies to assess the peri-implant tissue changes that occur over time using different treatment approaches, in terms of the timing of grafting procedures and of implant placement.

\section{ACKNOWLEDGMENTS AND DISCLOSURE STATEMENTS}

The authors report no conflicts of interest related to the present review.

\section{REFERENCES}

1. Schropp L, Wenzel A, Kostopoulos L, Karring T. Bone healing and soft tissue contour changes following singletooth extraction: a clinical and radiographic 12-month prospective study. Int J Periodontics Restorative Dent. 2003 Aug;23(4):313-23. [Medline: 12956475]

2. Lang NP, Pun L, Lau KY, Li KY, Wong MC. A systematic review on survival and success rates of implants placed immediately into fresh extraction sockets after at least 1 year. Clin Oral Implants Res. 2012 Feb;23 Suppl 5:39-66. [Medline: 22211305] [doi: 10.1111/j.1600-0501.2011.02372.x]

3. Hämmerle CH, Araújo MG, Simion M; Osteology Consensus Group 2011. Evidence-based knowledge on the biology and treatment of extraction sockets. Clin Oral Implants Res. 2012 Feb;23 Suppl 5:80-2. [Medline: 22211307] [doi: $10.1111 / \mathrm{j} .1600-0501.2011 .02370 . \mathrm{x}]$

4. Avila-Ortiz G, Chambrone L, Vignoletti F. Effect of alveolar ridge preservation interventions following tooth extraction: A systematic review and meta-analysis. J Clin Periodontol. 2019 Jun;46 Suppl 21:195-223. [Medline: 30623987] [doi: $10.1111 /$ jepe.13057] 
5. Vignoletti F, Matesanz P, Rodrigo D, Figuero E, Martin C, Sanz M. Surgical protocols for ridge preservation after tooth extraction. A systematic review. Clin Oral Implants Res. 2012 Feb;23 Suppl 5:22-38. [Medline: 22211304] [doi: $10.1111 / \mathrm{j} .1600-0501.2011 .02331 . \mathrm{x}]$

6. Avila-Ortiz G, Elangovan S, Kramer KW, Blanchette D, Dawson DV. Effect of alveolar ridge preservation after tooth extraction: a systematic review and meta-analysis. J Dent Res. 2014 Oct;93(10):950-8. [Medline: 24966231] [PMC free article: 4293706] [doi: 10.1177/0022034514541127]

7. Donos N, Mardas N, Chadha V. Clinical outcomes of implants following lateral bone augmentation: systematic assessment of available options (barrier membranes, bone grafts, split osteotomy). J Clin Periodontol. 2008 Sep;35(8 Suppl):173-202. [Medline: 18724850] [doi: 10.1111/j.1600-051X.2008.01269.x]

8. Raghoebar GM, Onclin P, Boven GC, Vissink A, Meijer HJA. Long-term effectiveness of maxillary sinus floor augmentation: A systematic review and meta-analysis. J Clin Periodontol. 2019 Jun;46 Suppl 21:307-318. [Medline: 30624789] [doi: 10.1111/jcpe.13055]

9. Benic GI, Bernasconi M, Jung RE, Hämmerle CH. Clinical and radiographic intra-subject comparison of implants placed with or without guided bone regeneration: 15-year results. J Clin Periodontol. 2017 Mar;44(3):315-325. [Medline: 27978603] [doi: 10.1111/jcpe.12665]

10. Benić GI, Jung RE, Siegenthaler DW, Hämmerle CH. Clinical and radiographic comparison of implants in regenerated or native bone: 5-year results. Clin Oral Implants Res. 2009 May;20(5):507-13. [Medline: 19302239] [doi: $10.1111 / \mathrm{j} .1600-0501.2008 .01583 . \mathrm{x}]$

11. Zumstein T, Billström C, Sennerby L. A 4- to 5-year retrospective clinical and radiographic study of Neoss implants placed with or without GBR procedures. Clin Implant Dent Relat Res. 2012 Aug;14(4):480-90. [Medline: 20491824] [doi: $10.1111 / \mathrm{j} .1708-8208.2010 .00286 . x$ ]

12. Schwarz F, Giannobile WV, Jung RE; Groups of the 2nd Osteology Foundation Consensus Meeting. Evidence-based knowledge on the aesthetics and maintenance of peri-implant soft tissues: Osteology Foundation Consensus Report Part 2-Effects of hard tissue augmentation procedures on the maintenance of peri-implant tissues. Clin Oral Implants Res. 2018 Mar;29 Suppl 15:11-13. [Medline: 29498125] [doi: 10.1111/clr.13109]

13. Liberati A, Altman DG, Tetzlaff J, Mulrow C, Gøtzsche PC, Ioannidis JP, Clarke M, Devereaux PJ, Kleijnen J, Moher D. The PRISMA statement for reporting systematic reviews and meta-analyses of studies that evaluate health care interventions: explanation and elaboration. J Clin Epidemiol. 2009 Oct;62(10):e1-34. [Medline: 19631507] [doi: $10.1016 /$ j.jclinepi.2009.06.006]

14. Hämmerle $\mathrm{CH}$, Chen ST, Wilson TG Jr. Consensus statements and recommended clinical procedures regarding the placement of implants in extraction sockets. Int J Oral Maxillofac Implants. 2004;19 Suppl:26-8. [Medline: 15635943]

15. Moher D, Liberati A, Tetzlaff J, Altman DG; PRISMA Group. Preferred reporting items for systematic reviews and meta-analyses: the PRISMA statement. J Clin Epidemiol. 2009 Oct;62(10):1006-12. [Medline: 19631508] [doi: 10.1016/j.jclinepi.2009.06.005]

16. Der Simonian R, Kacker R. Random-effects model for meta-analysis of clinical trials: an update. Contemp Clin Trials. 2007 Feb;28(2):105-14. [Medline: 16807131] [doi: 10.1016/j.cct.2006.04.004]

17. Kutkut A, Andreana S, Monaco E Jr. Clinical and radiographic evaluation of single-tooth dental implants placed in grafted extraction sites: a one-year report. J Int Acad Periodontol. 2013 Oct;15(4):113-24. [Medline: 24364175]

18. Kutkut A, Andreana S, Kim HL, Monaco E Jr. Extraction socket preservation graft before implant placement with calcium sulfate hemihydrate and platelet-rich plasma: a clinical and histomorphometric study in humans. J Periodontol. 2012 Apr;83(4):401-9. [Medline: 21861639] [doi: 10.1902/jop.2011.110237]

19. Heinemann F, Hasan I, Schwahn C, Bourauel C, Mundt T. Bone level change of extraction sockets with BioOss collagen and implant placement: a clinical study. Ann Anat. 2012 Nov;194(6):508-12. [Medline: 22244384] [doi: 10.1016/i.aanat.2011.11.012]

20. Barone A, Borgia V, Covani U, Ricci M, Piattelli A, Iezzi G. Flap versus flapless procedure for ridge preservation in alveolar extraction sockets: a histological evaluation in a randomized clinical trial. Clin Oral Implants Res. 2015 Jul;26(7):806-13. [Medline: 24580835] [doi: 10.1111/clr.12358]

21. Scheyer ET, Schupbach P, McGuire MK. A histologic and clinical evaluation of ridge preservation following grafting with demineralized bone matrix, cancellous bone chips, and resorbable extracellular matrix membrane. Int J Periodontics Restorative Dent. 2012 Oct;32(5):543-52. [Medline: 22754902]

22. Deli G, Petrone V, De Risi V, Tadic D, Zafiropoulos GG. Longitudinal implant stability measurements based on resonance frequency analysis after placement in healed or regenerated bone. J Oral Implantol. 2014 Aug;40(4):438-47. [Medline: 25106008] [doi: 10.1563/AAID-JOI-D-12-00014]

23. van Kesteren CJ, Schoolfield J, West J, Oates T. A prospective randomized clinical study of changes in soft tissue position following immediate and delayed implant placement. Int J Oral Maxillofac Implants. 2010 May-Jun;25(3):562-70. [Medline: 20556256]

24. Mahesh L, Narayan T, Kostakis G, Shukla S. Periotest values of implants placed in sockets augmented with calcium phosphosilicate putty graft: a comparative analysis against implants placed in naturally healed sockets. J Contemp Dent Pract. 2014 Mar 1;15(2):181-5. [Medline: 25095840] [doi: 10.5005/jp-journals-10024-1511] 
25. Felice P, Soardi E, Piattelli M, Pistilli R, Jacotti M, Esposito M. Immediate non-occlusal loading of immediate postextractive versus delayed placement of single implants in preserved sockets of the anterior maxilla: 4-month postloading results from a pragmatic multicentre randomised controlled trial. Eur J Oral Implantol. 2011 Winter;4(4):329-44. [Medline: 22282730]

26. Pang C, Ding Y, Zhou H, Qin R, Hou R, Zhang G, Hu K. Alveolar ridge preservation with deproteinized bovine bone graft and collagen membrane and delayed implants. J Craniofac Surg. 2014 Sep;25(5):1698-702. [Medline: 25148644] [doi: 10.1097/SCS.0000000000000887]

27. Tallarico M, Xhanari E, Pisano M, Gatti F, Meloni SM. Molar replacement with 7 mm-wide diameter implants: to place the implant immediately or to wait 4 months after socket preservation? 1 year after loading results from a randomized controlled trial. Eur J Oral Implantol. 2017;10(2):169-178. [Medline: 28555207]

28. Tallarico M, Xhanari E, Pisano M, De Riu G, Tullio A, Meloni SM. Single post-extractive ultra-wide 7 mm-diameter implants versus implants placed in molar healed sites after socket preservation for molar replacement: 6-month postloading results from a randomised controlled trial. Eur J Oral Implantol.2016;9(3):263-275. [Medline: 27722224]

29. Cardaropoli D, Tamagnone L, Roffredo A, Gaveglio L. Evaluation of Dental Implants Placed in Preserved and Nonpreserved Postextraction Ridges: A 12-Month Postloading Study. Int J Periodontics Restorative Dent. 2015 SepOct;35(5):677-85. [Medline: 26357697] [doi: 10.11607/prd.2309]

30. Barone A, Orlando B, Cingano L, Marconcini S, Derchi G, Covani U. A randomized clinical trial to evaluate and compare implants placed in augmented versus non-augmented extraction sockets: 3-year results. J Periodontol. 2012 Jul;83(7): 836-46. [Medline: 22141358] [doi: 10.1902/jop.2011.110205]

31. Marconcini S, Giammarinaro E, Derchi G, Alfonsi F, Covani U, Barone A. Clinical outcomes of implants placed in ridgepreserved versus nonpreserved sites: A 4-year randomized clinical trial. Clin Implant Dent Relat Res. 2018 Dec;20(6): 906-914. [Medline: 30307130] [doi: 10.1111/cid.12682]

32. Felice P, Pistilli R, Barausse C, Trullenque-Eriksson A, Esposito M. Immediate non-occlusal loading of immediate postextractive versus delayed placement of single implants in preserved sockets of the anterior maxilla: 1-year post-loading outcome of a randomised controlled trial. Eur J Oral Implantol. 2015 Winter;8(4):361-72. [Medline: 26669546]

33. Esposito M, Barausse C, Pistilli R, Jacotti M, Grandi G, Tuco L, Felice P. Immediate loading of post-extractive versus delayed placed single implants in the anterior maxilla: outcome of a pragmatic multicenter randomised controlled trial 1-year after loading. Eur J Oral Implantol. 2015 Winter;8(4):347-58. [Medline: 26669545]

34. Esposito M, Zucchelli G, Cannizzaro G, Checchi L, Barausse C, Trullenque-Eriksson A, Felice P. Immediate, immediatedelayed (6 weeks) and delayed (4 months) post-extractive single implants: 1-year post-loading data from a randomised controlled trial. Eur J Oral Implantol. 2017;10(1):11-26. [Medline: 28327692]

35. Sanz-Sánchez I, Ortiz-Vigón A, Sanz-Martín I, Figuero E, Sanz M. Effectiveness of Lateral Bone Augmentation on the Alveolar Crest Dimension: A Systematic Review and Meta-analysis. J Dent Res. 2015 Sep;94(9 Suppl):128S-42S. [Medline: 26215467] [doi: 10.1177/0022034515594780]

36. Sanz-Sánchez I, Carrillo de Albornoz A, Figuero E, Schwarz F, Jung R, Sanz M, Thoma D. Effects of lateral bone augmentation procedures on peri-implant health or disease: A systematic review and meta-analysis. Clin Oral Implants Res. 2018 Mar;29 Suppl 15:18-31. [Medline: 29498126] [doi: 10.1111/clr.13126]

37. Mello CC, Lemos CAA, Verri FR, Dos Santos DM, Goiato MC, Pellizzer EP. Immediate implant placement into fresh extraction sockets versus delayed implants into healed sockets: A systematic review and meta-analysis. Int J Oral Maxillofac Surg. 2017 Sep;46(9):1162-1177. [Medline: 28478869] [doi: 10.1016/i.ijom.2017.03.016]

38. Mangano FG, Mastrangelo P, Luongo F, Blay A, Tunchel S, Mangano C. Aesthetic outcome of immediately restored single implants placed in extraction sockets and healed sites of the anterior maxilla: a retrospective study on 103 patients with 3 years of follow-up. Clin Oral Implants Res. 2017 Mar;28(3):272-282. [Medline: 26913807] [doi: 10.1111/clr.12795]

39. Raes S, Eghbali A, Chappuis V, Raes F, De Bruyn H, Cosyn J. A long-term prospective cohort study on immediately restored single tooth implants inserted in extraction sockets and healed ridges: CBCT analyses, soft tissue alterations, aesthetic ratings, and patient-reported outcomes. Clin Implant Dent Relat Res. 2018 Aug;20(4):522-530. [Medline: 29671940] [doi: 10.1111/cid.12613]

40. Salvi GE, Monje A, Tomasi C. Long-term biological complications of dental implants placed either in pristine or in augmented sites: A systematic review and meta-analysis. Clin Oral Implants Res. 2018 Oct;29 Suppl 16:294-310. [Medline: 30328184] [doi: 10.1111/clr.13123] 


\section{To cite this article:}

Ramanauskaite A, Borges T, Almeida BL, Correia A.

Dental Implant Outcomes in Grafted Sockets: a Systematic Review and Meta-Analysis

J Oral Maxillofac Res 2019;10(3):e8

URL: http://www.ejomr.org/JOMR/archives/2019/3/e8/v10n3e8.pdf

doi: $\underline{10.5037 / \text { jomr.2019.10308 }}$

Copyright (C) Ramanauskaite A, Borges T, Almeida BL, Correia A. Published in the JOURNAL OF ORAL \& MAXILLOFACIAL RESEARCH (http://www.ejomr.org), 5 September 2019.

This is an open-access article, first published in the JOURNAL OF ORAL \& MAXILLOFACIAL RESEARCH, distributed under the terms of the Creative Commons Attribution-Noncommercial-No Derivative Works 3.0 Unported License, which permits unrestricted non-commercial use, distribution, and reproduction in any medium, provided the original work and is properly cited. The copyright, license information and link to the original publication on (http://www.ejomr.org) must be included. 